Sādhanā Vol. 40, Part 1, February 2015, pp. 263-276. (C) Indian Academy of Sciences

\title{
Quantitative study on crack of meso-damage and fracture concrete based on CT technique
}

\author{
WEI TIAN ${ }^{1,3, *}$, FANING DANG ${ }^{2}$ and YONGLI XIE ${ }^{3}$ \\ ${ }^{1}$ School of Civil Engineering, Chang' an University, Xi' an 710061, China \\ ${ }^{2}$ Institute of Geotechnical Engineering, Xi' an University of Technology, \\ Xi'an 710048, China \\ ${ }^{3}$ School of Highway, Chang' an University, Xi' an 710064, China \\ e-mail: tianwei@chd.edu.cn
}

MS received 27 June 2014; revised 17 September 2014; accepted 20 September 2014

\begin{abstract}
The meso-mechanics experiment of concrete specimen is carried out by means of CT technology, the whole degradation deformation process of meso-cracks generation, propagation, coalescence and failure is obtained under uniaxial compression condition. Based on CT image of concrete meso-damage evolution, the recognition and extraction of meso-cracks are realized by means of the digital imageprocessing (DIP) technique, and the basic geometrical characteristics of meso-crack patterns are statistically analysed from length, width and area. The quantitative analysis of the meso-fracture process of concrete materials is performed. The results demonstrate that the quantitative analysis of the internal meso-crack of concrete can be taken as a quantitative index to reflect the damage and fracture process thereby the meso-fracture mechanism of concrete material is thoroughly investigated. So it has brought forth some new ideas to the study of damage evolution law of concrete under uniaxial compression condition.
\end{abstract}

Keywords. Concrete; meso-damage evolution; CT image; meso-crack; quantitative analysis.

\section{Introduction}

Concrete is a highly heterogeneous, multiphase composite material. On the mesoscale view, concrete is often considered to be a three-phase composite structure. It is a mixture of aggregate, mortar and porosity. So the strength, deformation and failure of concrete are related to the properties of the meso-structure composite structure.

Therefore, to explain the macroscopic mechanical properties of concrete materials and build the internal structure association with macroscopic properties, the generation and propagation of

${ }^{*}$ For correspondence 
meso-cracks are studied under the different stress state. In fact, the failure on the macro scale is the result of the accumulation and development of damage and cracks at the meso-level.

$\mathrm{X}$-ray computed tomography is a non-destructive testing method with the ability of threedimension meso-structure investigation and it can realize the continuous observation of the sample at all stage. So it has brought forth a new idea to the study of the whole degradation deformation process of meso-cracks generation, propagation and failure. Morgan et al (1980) used the concrete medical CT to scan the concrete specimens for the first time and obtained the clear cross-sectional images of aggregate, mortar and crack. Impregnation method was used to realize the quantitative analysis of micro cracks of concrete material (Ammouche et al 2000). The whole CT images of meso-cracks generation, propagation, and failure of the concrete specimen was obtained under the uniaxial compression condition in real time Chen et al (2006). Wong $\&$ Chau (2005) estimated the air void and aggregate distribution in normal and high-strength concrete specimens in various loading cases using CT. A 3D concrete specimen was reconstructed using CT images, and a damage parameter was identified (Wang et al 2007), and Suzuki et al (2010) used the same method to evaluate damage in freeze-thawed concrete (Garboczi 2002) combined X-ray tomography with spherical harmonics and subsequently analysed the shape of concrete particles.

The problems that it was difficult to determine whether the crack appears or not and specific region of the crack were discussed theoretically. So based on CT images of static compression under the real time scanning, damage-fracture divisional zones theory was applied to analyse concrete CT images. Then the divisional criteria of the divisional zones from the CT images was discussed and determined, the CT data information on these divisional zones at each loading stage was analysed Dang et al (2007). According to CT physical principle and the difference image algorithm, the method of quantitative analysis about the slit region in the concrete CT images (Ding et al 2006). The calculation method of crack's width was also presented (Ding et al 2009). Based on CT data and damaged evolution, Tian et al (2010) studied the meso-damage characteristics of concrete under uniaxial compression used fractal theory, and support vector machine (SVM) theory (Tian et al 2011).

In this paper, we take the CT images of concrete meso-damage evolution under the uniaxial compression condition as the research object, put CT number variation of each pixel point in the image as judgement basis, the new digital image analysis methods are developed and the recognition and extraction of meso-cracks are realized by means of the digital-image-processing (DIP) technique. The basic geometrical characteristics of meso-crack patterns are statistically analysed from length, width and area. So the crack zone conducts were quantitatively analysed. Thereby it not only provides a feasible method for the research on the damage mechanism of concrete, but also creates a new way for exploring the damage evolution of concrete material under the uniaxial compression condition.

\section{Experimentation}

\subsection{Materials and specimen preparation}

The cylindrical specimens of $60 \mathrm{~mm}$ diameter and $120 \mathrm{~mm}$ in height are drilled out from cube specimens, concrete grade: $\mathrm{C} 20$, water cement ratio: 0.5 , aggregate specifications: natural crushed stone and natural river sand. The maximum diameter of grain size is up to $10 \mathrm{~mm}$. The core specimens are inspected with CT scans at RenHe Medical Center, Three Gorge University. The measurement conditions are summarized in table 1. 
Table 1. Setting used for helical CT scan.

\begin{tabular}{lc}
\hline Parameter & Index \\
\hline Helical CT machine & PHILIPS brilliance spiral CT \\
Speed & $0.4 \mathrm{~s} /$ rotation \\
Exposure & $120 \mathrm{kw}$ and $300 \mathrm{~mA}$ \\
Recon matrix & $512 * 512$ \\
Density differentiating rate & $0.3 \%$ \\
Slice thickness & $0.5 \mathrm{~mm}$ \\
\hline
\end{tabular}

The loading equipment used in this study is newly developed portable dynamic loading equipment (PDLE) which can allow image acquisition without unloading the specimen Loading path: load controlling, loading rate $0.2 \mathrm{KN} / \mathrm{s}$, add to $60 \mathrm{KN}$. Stop loading, not to change displacement and carry on the second scan for the concrete specimen. Then control the displacement and loading rate of $0.002 \mathrm{~mm} / \mathrm{s}$. By analogy, test stops when the specimen is destroyed, and the failure load is up to $101.98 \mathrm{KN}$. Photographs of the specimen are shown in figure 1. The structure of the PDLE is shown in figures 2 and 3.

\subsection{Sample testing}

The CT number in Hounsfield Units represents the mean X-Ray absorption associated with each area on the CT image. The CT number varies according to the material properties, generally adjusted to 0 for water and to -1000 for air. In this experiment, at cross section of concrete, the average CT numbers are between 2400 and 2560. The specimen is scanned constantly at $0.5 \mathrm{~mm}$ pitch overlapping. A total of $3862 \mathrm{D}$-images are obtained from each specimen, so we give a representative specimen to scan and analyse. We choose the specimen labelled CONC010 used in this test. The complete stress-strain curve is obtained during testing of the specimen in figure 4 . Here the number in the figure shows the 10-time scanning sequence points. The curve of average CT numbers-strain of CONC010 is shown in figure 5.

The results obtained are illustrated in the following. The crack evolution process of concrete is shown in figures 6 and 7. According to the figures, the damage process of concrete can be divided into five stages:

(i) The consolidation stage: At the beginning of the experiment, the average CT number of each section changes minimally. With load increasing, the average CT number of each section indicates increasing tread. Because the density of a concrete specimen increases with the volume of some meso-cracks and pores are compressed and strength also increases.

(ii) The meso-crack stable propagation stage: With the increase of load, the average CT numbers begin to decrease in each scanning section until the stress is up to $33.19 \mathrm{MPa}$, which is an important characteristic. It shows that mesodamage begins to propagate through the cement paste and along the interfacial transition zone (ITZ). These cracks usually generate from the pore boundaries and propagate in the directions of maximum compression.

(iii) The volume enlarge stage: The density of specimen decreases but does not shown obvious change in CT images. The average CT numbers have small change with load. The human eyes have lower resolution to gray colour so it is difficult to find CT scale cracks in the gray zone of CT image. It appears that the accumulation process is dependent on the number of cracks at a certain stress level. 

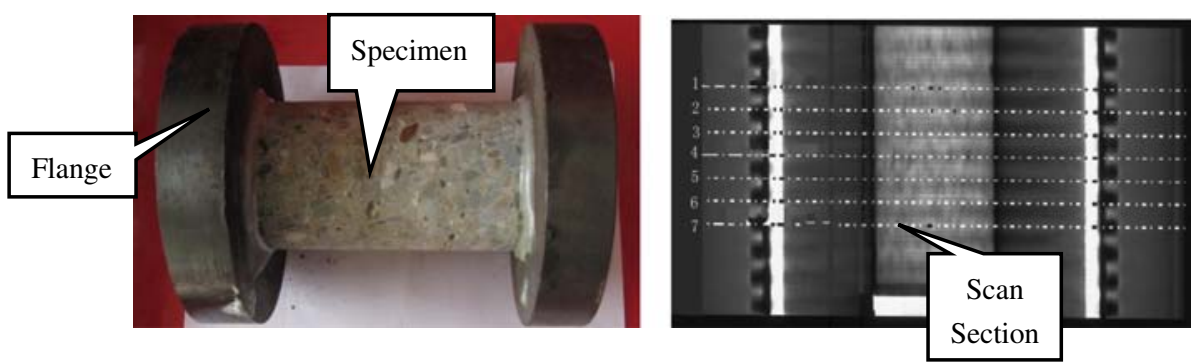

Figure 1. Geometry of the experimental concrete specimen.

(iv) The meso-crack unstable propagation stage: When stress is up to $36.08 \mathrm{MPa}$ (the peak load), the average CT numbers reduce rapidly in each scanning section. The crack gradually enlarges as the average CT numbers sharply decrease so that the density of specimen is becoming more and more inclined to decrease. The volume of each scanning section of specimen has significant expansion. But it is hard to differentiate by naked eye.

(v) The finally completed fracture stage: When the axial stress is from $32.22 \mathrm{MPa}$ to $12.71 \mathrm{MPa}$, specimen enters the damage stage. At this time the average CT numbers in scanning section drastically decreased, which indicates that strength drops apparently and specimens have the rapid expansion. Further, these meso-cracks propagate and converge rapidly and the width more and more widen. Finally, the macro principal cracks form. At the same time the specimen is completely fractured.

In the static loading case, cracks remain around the aggregate and the path of the cracks is curved. When the specimen is fractured, a main crack that cut the specimen into two parts is observed (see figure 7e). Some aggregates also emerge from the failure surface. The surface of these aggregates is rough and with no lustre, and some cement mortars adhere to it (see figure 8), indicating that the aggregate is not cut.

\section{Results and discussion}

\subsection{The quantitative analysis of test image}

In this paper, the $\mathrm{CT}$ image is produced by the $\mathrm{CT}$ testing allowed us to employ an extensive library of image analysis. The CT image of concrete contains a large number of image

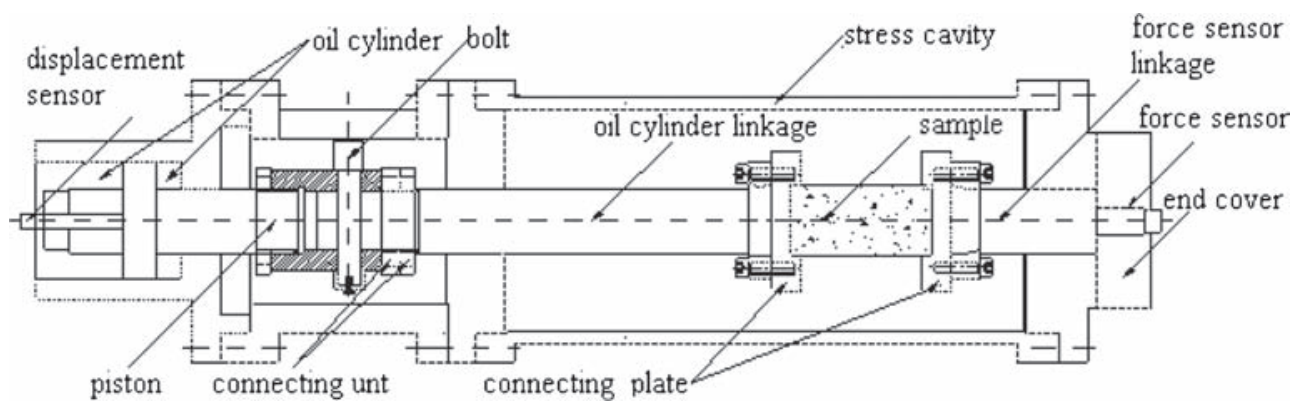

Figure 2. Sketch of portable dynamic loading equipment. 


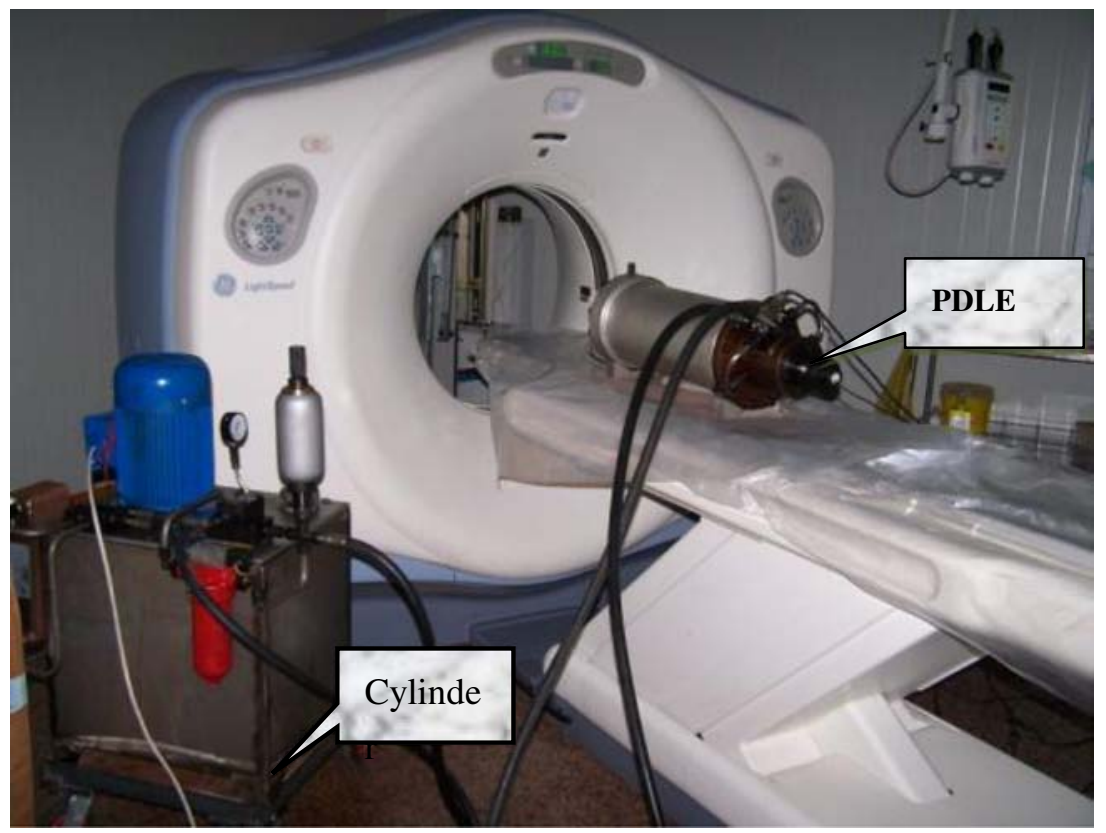

Figure 3. Scanning process.

information about meso-structure. It has the vital significance for the analysis of CT testing and how to extract the effective information about the concrete material mesostructure changed in the loading process. We tried to dig out and extract the valuable information of the meso-crack in concrete by studying the change of CT numbers in scanning section.

So the CT image information of the meso-crack in concrete has helped in quantitative analysis. Meanwhile, the concrete meso-damage fracture mechanism is deeply investigated.

It can be seen from figures 9 and 10 that different grey levels corresponding to various CT numbers, as well as the different CT numbers associated with the density of internal concrete

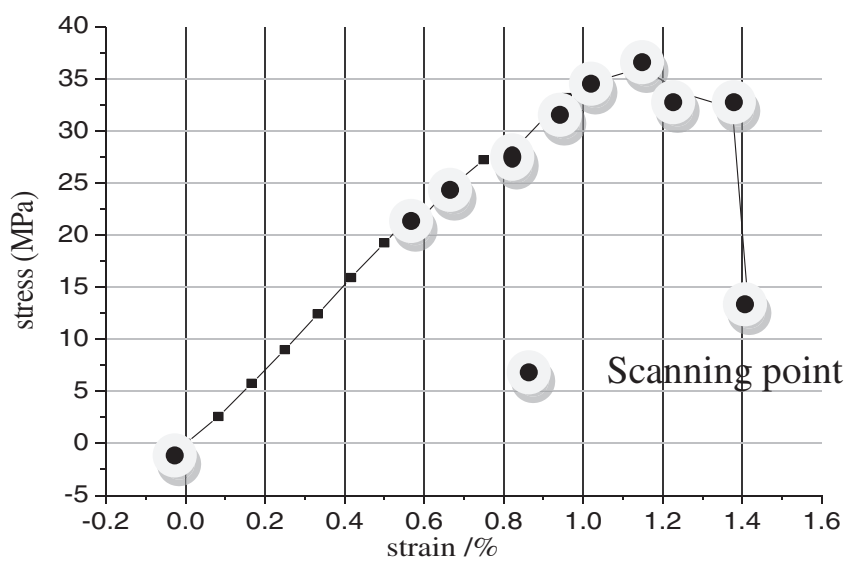

Figure 4. Stress-strain curve. 


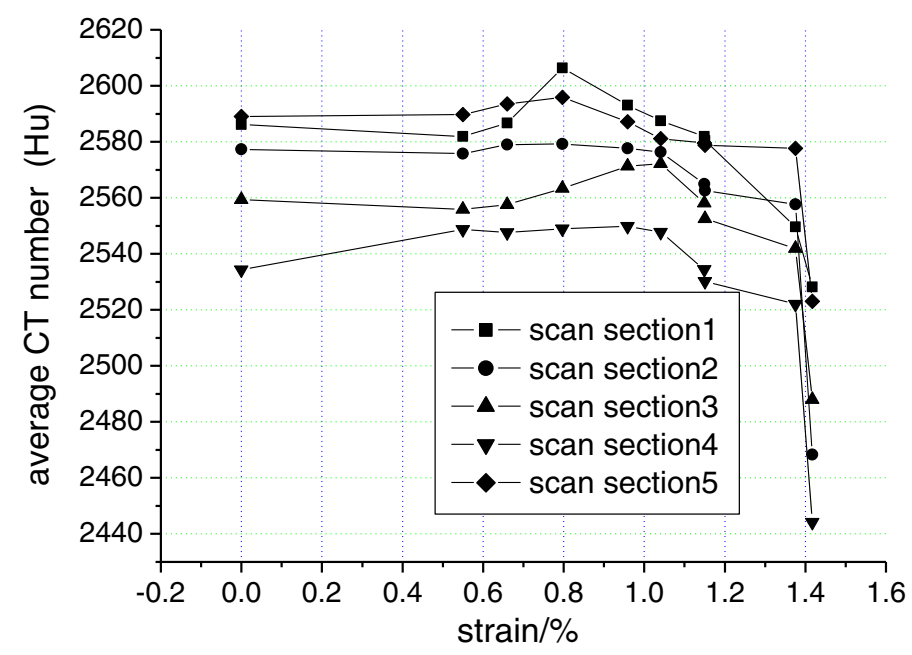

Figure 5. The average CT numbers-strain curve.

material. For example, along the blue lines, the CT number of the aggregate is large and the colour in the CT image is close to white. By contrast, the CT numbers of the cracks or pores are very small and the colour in the CT image is black or nearly black. Cement mortar is found around the aggregates and the density of this region is lower than that of the aggregates but larger than that of the cracks and pores. Thus, its colour is light or dark grey.

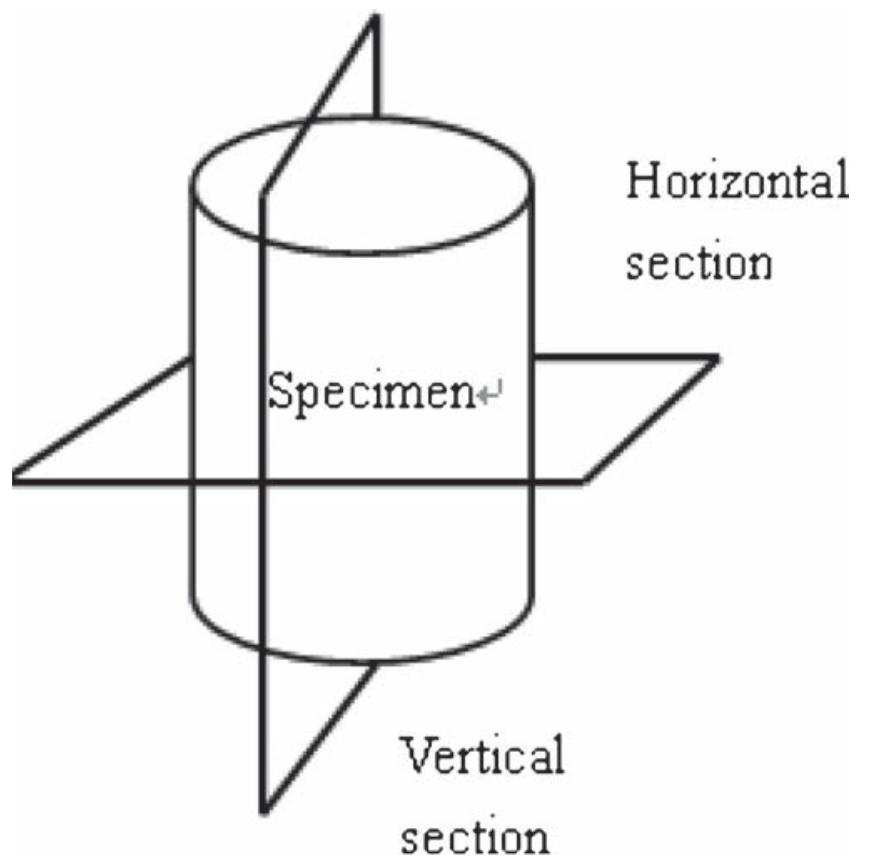

Figure 6. Illustration of specimen volume and different section. 
Horizontal section

Vertical section

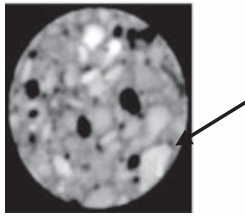

Concrete material is homogeneous. The meso-structure defects are seen from the horizontal cross section and vertical section. This kind of defect is randomly distributed.

$\sigma=0.00 \mathrm{MPa}$

(a) Unload stage

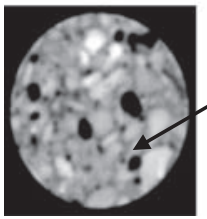

$\sigma=24.59 \mathrm{MPa}$

By average CT numbers, the initial defects (damage) including holes and meso-cracks of concrete specimen close in the compression process. Specimens are compacted.

(b) Consolidation stage
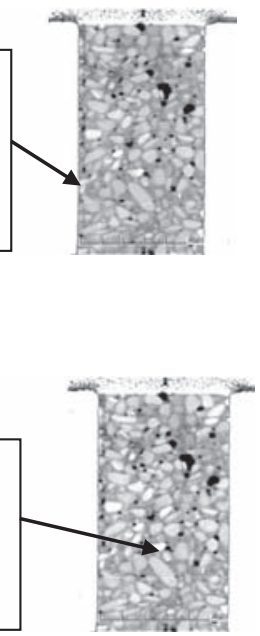$$
\text { ( }
$$

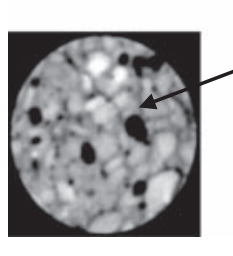

$\sigma=33.19 \mathrm{MPa}$

The internal defect zone of specimen first appears mesocracks due to the local tensile stress concentration. Meanwhile the meso-damage begins to propagate along the ITZ.

(c) Meso -crack stable propagation stage
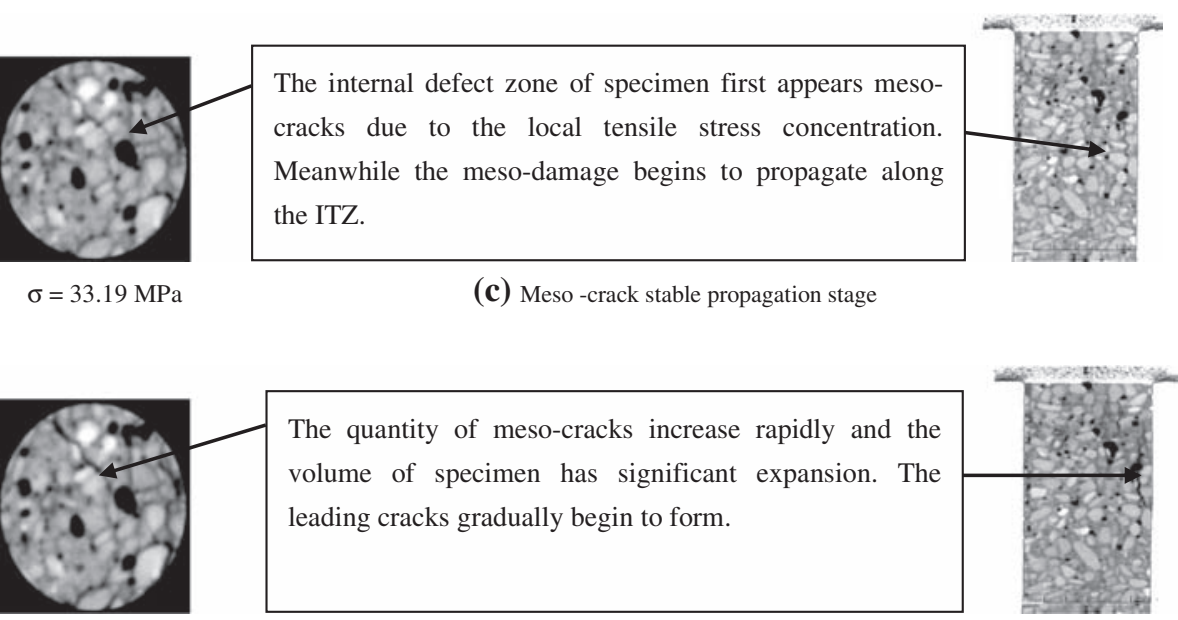

The quantity of meso-cracks increase rapidly and the volume of specimen has significant expansion. The leading cracks gradually begin to form.

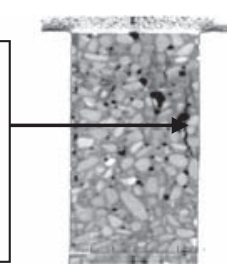

$\sigma=36.08 \mathrm{MPa}$

(d) Meso-crack unstable propagation stage

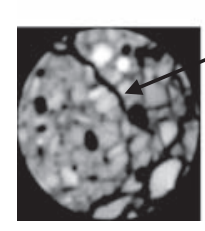

$\sigma=12.7 \mathrm{MPa}$
Meso-cracks in cross section sharply extend along the loading direction, the macroscopic cracks are obvious and the specimen is completely fractured.

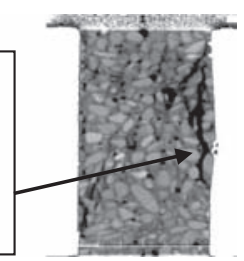

(e) Finally fractured stage

Figure 7. The meso-structure damage of concrete specimen under different axial stresses. 


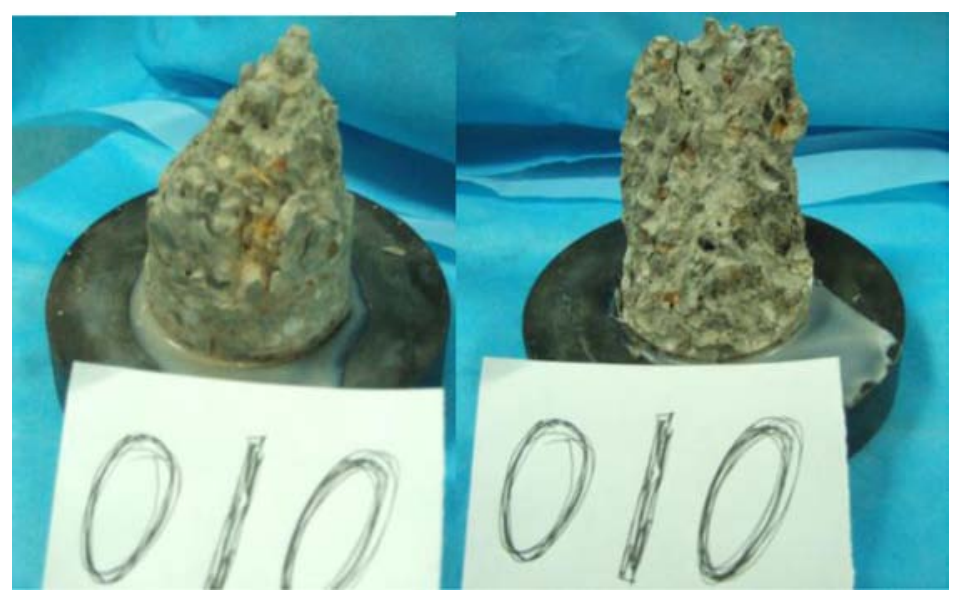

Figure 8. Digital picture of concrete failure.

\subsection{The method of image processing}

The paper uses MATLAB and ENVI software to process image and analyse quantitatively. Before segmentation of the image, some treatments such as smoothing and island removing are carried out to remove the noise so that extract more testing information of the cracks and make the edges more highlighted.

\subsection{The extraction of CT image of crack}

3.3a The extraction methods of crack: The density variation of neighbouring points can measure whether the point belongs to the crack zone or not. Because of the initial damage of concrete it is hard to extract cracks according to the absolute CT number. Meanwhile, a single point of CT number is difficult to obtain with the change of the loading process, so that the error is very large. Crack is irreversible once created and the extension trend does not decrease in the loading process, it can change the CT number distribution in the scanning section. But the CT number's

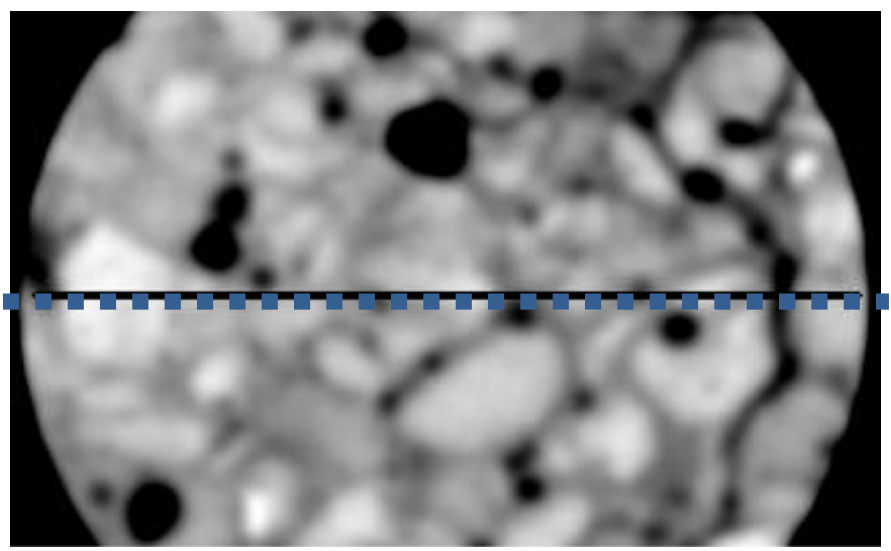

Figure 9. The enlarged part of CT image. 


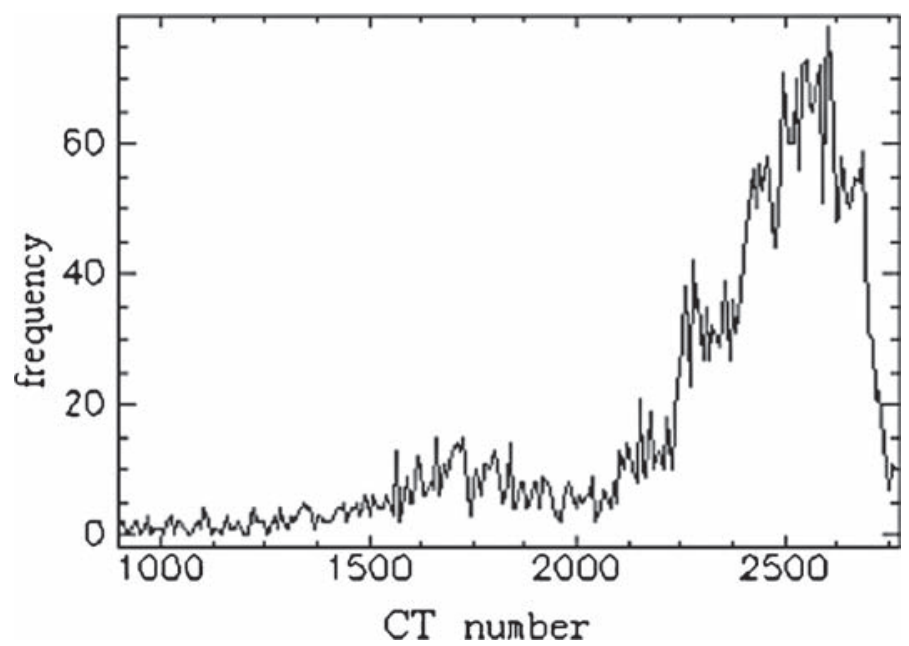

Figure 10. CT number variation along the line.

variation of each pixel point can accurately reflect the influence of material mechanics deterioration. Now the CT number's variation of each pixel point and the criterion of crack are defined, as follows

$$
\begin{gathered}
\Delta H_{i, j}=\max \left(\Delta H_{i-(i+1), j}, \Delta H_{i, j-(j+1)}\right) \\
\Delta H_{i-(i+1), j}=\left|H_{i, j}-H_{i+1, j}\right|, \Delta H_{i, j-(j+1)}=\left|H_{i, j}-H_{i, j+1}\right|,
\end{gathered}
$$

$\mathrm{H}_{\mathrm{i}, \mathrm{j}}$ is the CT number of its row and its column , $\mathrm{i}=1,2, \cdots, 512=1,2, \cdots, 512$.

Image of Row 512 and Column 512 is at the edge, away from the crack zone and scanning zone. So define $\Delta \mathrm{H}_{\mathrm{i}, \mathrm{j}}=0$, namely it has no effect on crack extraction. According to the above formula, the binarization processing of crack image is developed under certain crack extraction threshold value $\xi(\mathrm{ETV})$.

where

$$
\begin{gathered}
\Delta \mathrm{H}_{\mathrm{i}, \mathrm{j}}<\xi \text { non }- \text { crackzone. } \\
\Delta \mathrm{H}_{\mathrm{i}, \mathrm{j}} \geq \xi, \text { crackzone. }
\end{gathered}
$$

3.3b The extraction of crack: In order to determine the threshold $\xi$, taking the CT images with $\sigma=12.7 \mathrm{MPa}$ as an example, the threshold value $\xi$ and the total pixels of crack is drawn under $\xi=0.1 \sim 0.9$ by 0.05 continuous increasing. It is shown in figure 11 .

According to figure 11, the total pixels of crack sharply reduce as the threshold values $\xi$ increase gradually. Curve down slowly after the threshold value is greater than 0.5 . It demonstrates that the analysis of targets gradually focuses on the crack zone, and undamaged zone influence on the crack zone is disappeared step-by-step. After the linear fitting of the start and end of curve, the intersection point of curve $(\xi=0.48)$ can be an extraction threshold value of crack. The CT images of scanning section cracks are extracted under different loading phase by the CT numbers' variation of each pixel point and adjacent pixel points and extraction criterion of crack. The binary image containing cracks is received and shown in figure 12. Only three binary images of scanning sections are listed during the loading phase to demonstrate. 


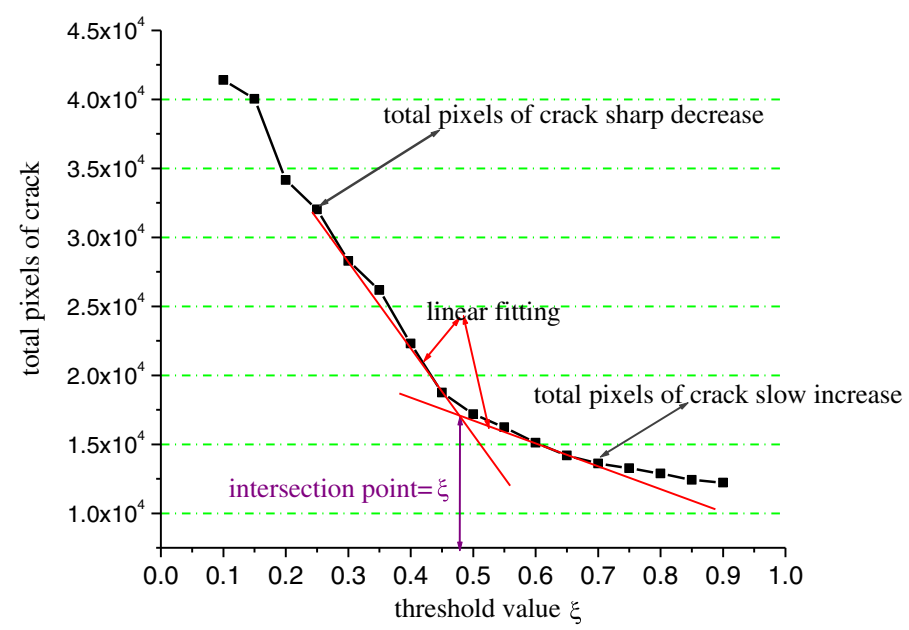

Figure 11. Curve of $\xi$ and the total pixels of crack.

An example of image segmentation is illustrated in figure 12, where the black and white image of figure $12 \mathrm{~b}$ is created from the grayscale image of figure $12 \mathrm{a}$, by setting all pixel values less than the threshold equal to zero (black), and all values greater than the threshold to one (white). In this manner, generated cracks and pores can be easily identified.

The binary image shown in figure 12 includes small isolated pores, which are eliminated by the application of a minimum size threshold value for objects of 10 pixels. A skeletonized binary image is obtained by binary thinning. For every thinning step, pixels that are not relevant to the connectivity of an object are removed from the object margins, this process continues until all objects are reduced to a width of one pixel that approximates the skeleton. An example of a binary-thinned image of crack is presented in figure 13 .

\subsection{Quantitative analysis of meso- cracks}

After image processing, the quantitative information is further analysed and calculated. According to the characteristics of the meso-damage of concrete in the CT image, the geometrical characteristics of meso-crack patterns are quantitatively analysed from the length, width and area.

3.4a The length and width of crack: The total pixels of curve are counted after refinement of crack, and the length of crack is calculated through the image resolution. Table 2 shows that the length of meso-crack gradually increases from $64.5 \mu \mathrm{m}$ to $492.07 \mu \mathrm{m}$ with the increase of stress. When the stress is 36.08 to $32.22 \mathrm{MPa}$, the crack's length of scanning section increases $44 \%$ and the width $54 \%$. At this time, the length and width of crack develop faster, and it is the fastest damage evolution stage. When the stress is $12.71 \mathrm{MPa}$, the length growth of crack reaches the maximum $70.1 \%$, the width has reached $78.1 \%$. It indicates that the meso- cracks have propagated and converged, the macro-crack is formed, so the specimen is completely fractured.

3.4b The area of crack: The area of crack is the product of the number of units that belong to the crack zone and distinguish units' zone. According to the basic data of meso-crack area, 

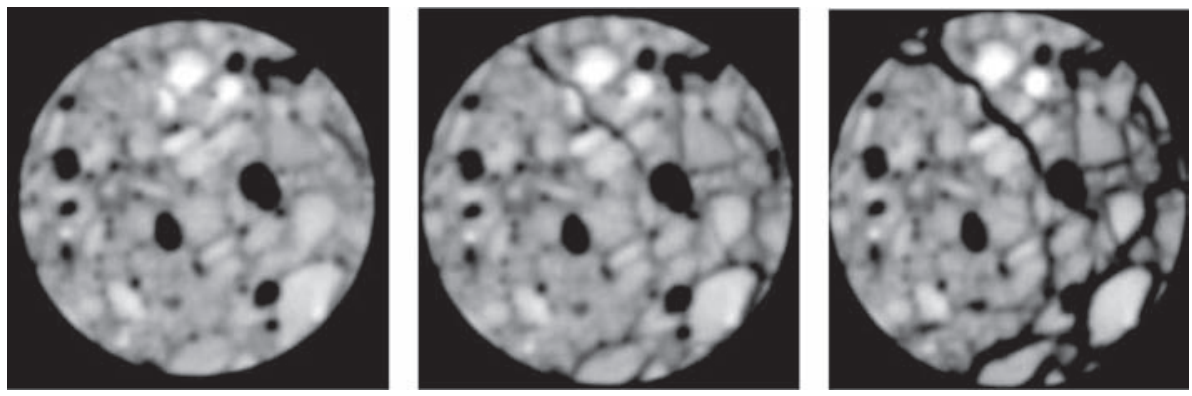

(a) Gray scale image
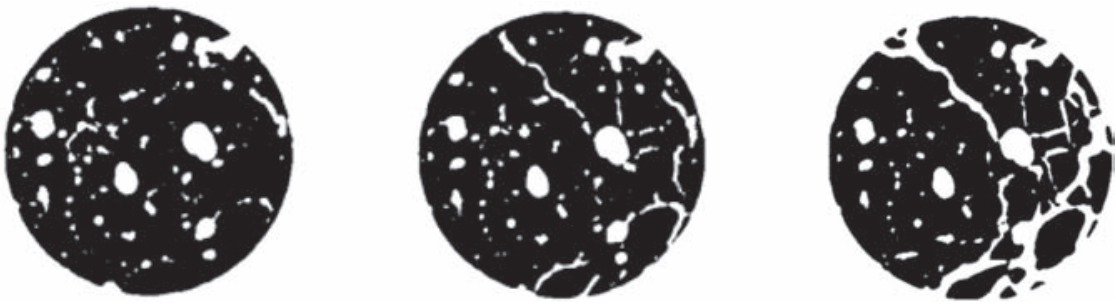

(b) Binary image segmentation

Figure 12. Image-binarizing processing.

when the stress is from 36.08 to $32.22 \mathrm{MPa}$, total area of the meso-cracks in scanning section increases at a faster pace. Especially when the stress is $32.22 \mathrm{MPa}$, the total area of the crack growth is up $63 \%$. Compared with the previous loading, the meso-cracks are developed rapidly under the loading.

When the stress is $12.71 \mathrm{MPa}$, the growth of the crack total area in scanning section reaches the maximum number. The performance in energy dissipative of macrostructure failure is mainly presented as generation and propagation of meso-cracks, namely the increase of the total area of the meso-cracks. So, it is suggested that the cracks grow along the weakness plane of concrete material.
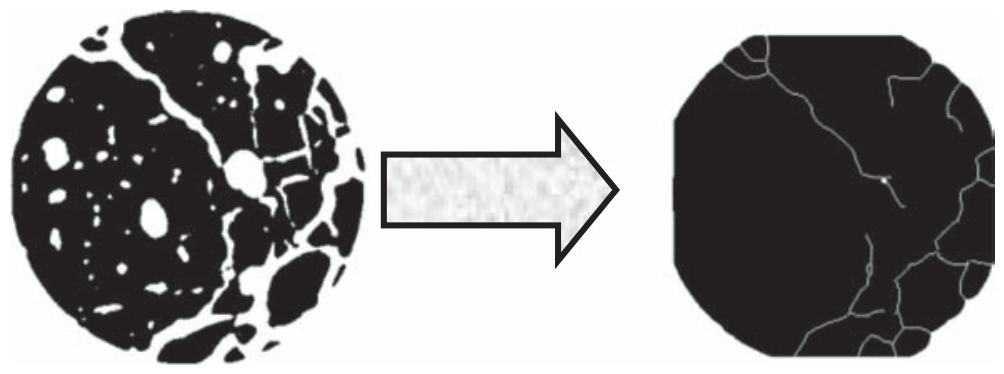

Figure 13. binary-thinned image of the crack. 
Table 2. Statistical results of meso-cracks region.

\begin{tabular}{lcccc}
\hline$\sigma / \mathrm{MPa}$ & Average CT Number $/ \mathrm{HU}$ & $\mathrm{Lc} / \mu \mathrm{m}$ & $\mathrm{Wc} / \mu \mathrm{m}$ & $\mathrm{Sc} / \mu \mathrm{m}^{2}$ \\
\hline 33.19 & 2302.20 & 64.5 & 9.80 & 9 \\
36.08 & 2262.05 & 149.6 & 19.43 & 12 \\
33.61 & 2065.94 & 214.9 & 30.10 & 82 \\
32.22 & 1978.15 & 288.9 & 58.59 & 133.4 \\
12.71 & 1770.46 & 492.07 & 104.37 & 277.8
\end{tabular}

Where $\mathrm{Lc}=$ total crack length in a section $(\mu \mathrm{m}), \mathrm{Wc}=$ total crack width in a section $(\mu \mathrm{m}), \mathrm{Sc}=$ total crack surface area in a section $\left(\mu \mathrm{m}^{2}\right)$.

\subsection{Image reconstruction}

The 3D internal structures of the concrete spatial distribution of aggregate, mortar and porosity can be acquired using the X-ray CT scanning technique. Several cross-section images of concrete specimen have been acquired using CT system can be put together and rendered to produce a volume image. The image reconstruction work is carried out with mimic software. Threedimensional renderings of CT data are shown in figure 14a. The reconstructed 3D image of the scanned of crack zone is shown in figure 14b. It can be clearly seen that cracks from different angles cracks branching and bridging. The internal crack growth can be clearly observed in three-dimension and the critical features of fracture are clearly illustrated.

It lays a foundation not only for further analysis of the regularity on the evolution of crack, but also quantitative determination of the shapes of crack zone.

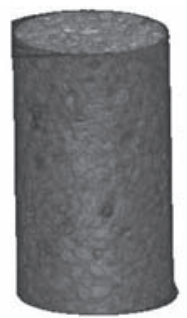

3D specimen reconstruction

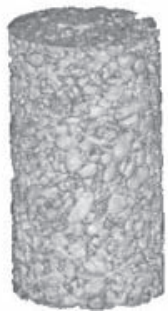

$3 \mathrm{D}$ aggregate reconstruction

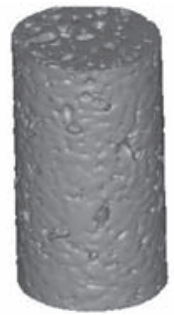

3D mortar reconstruction

(a)
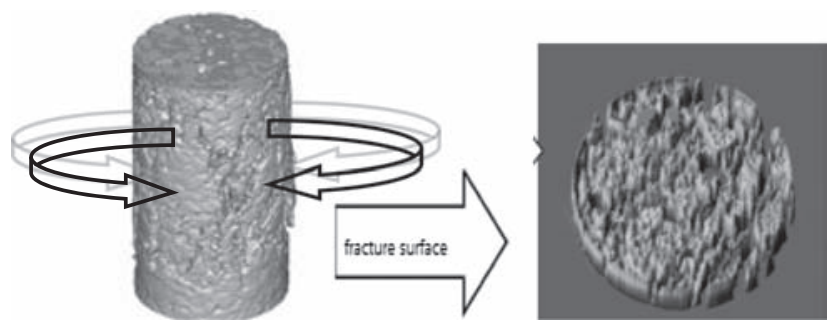

(b)

Figure 14. 3D concrete reconstruction. (a) 3-Dspeciment reconstruction (left), 3D aggregate reconstruction (middle) and 3D mortar reconstruction (right); b) 3-D crack reconstruction of concrete. 


\section{Conclusions}

In this paper, the whole process of the concrete material mesodamage fracture evolution is carried out on the quantitative test by $\mathrm{CT}$ scanning technique. Meanwhile, the propagation images of meso-cracks are obtained under the uniaxial compression condition. Furthermore, the evaluation law of the meso-crack during the deformation and fracture of concrete is disclosed by the research of mesomechanics. The conclusions drawn are summarized below.

(i) According to the definition of $\Delta \mathrm{H}_{\mathrm{i}, \mathrm{j}}=0$, the extraction threshold value for crack zone is determined, and the binary image only containing crack is achieved by image processing technology (DIP). The meso-cracks geometrical characteristics including length, width and area of meso-cracks are extracted from binary image. So, the quantitative description of the dynamic evolution process of the crack is realized.

(ii) The quantitative analysis of internal meso-cracks can be regarded as the quantity index to reflect the damage process of concrete. It confirms that the failure process of the concrete is gradually generated, which is transition step-by-step from the meso-damage to the macrofracture. In the static loading cases, a consolidation stage in the experimental process is observed and an obvious crack undeveloped stage occurs before the complete damage stage. In this stage, the average CT numbers barely changed. Cracks can be developed all along with load and the average CT number is gradually decreased. Then, the meso-cracks have propagated and converged rapidly. In the end, the leading macrocracks begin to form. A main crack that cuts through the specimen is observed and specimen is broken into pieces. These results will provide some helpful information to investigate the concrete meso-damage fracture mechanism.

\section{Acknowledgements}

This work was financially supported by the National Natural Science Foundation of China (Grant No.51009010, 51379015), China Postdoctoral Science Foundation funded project (Grant No. 2012M521727), and the Special Fund for Basic Scientific Research of Central College, Chang'an university (Grant No.CHD2012ZD014), and the Postdoctoral Science Foundation funded project of Shaanxi Provincial.

\section{References}

Ammouche A, Breysse D and Hornain H 2000 A new image analysis technique for the quantitative assessment of micro cracks in cement-based materials. Cement Concrete Research 30(6): 25-35

Chen H-Q, Ding W-H and Dang F-N 2006 Real time observation on meso fracture process of concrete using X-ray CT under uniaxial compressive condition. J. Hydraul. Eng. 37(9): 1044-1050

Dang F-N, Liu Y-W and Ding W-H 2007 Quantitative Analysis of concrete CT image Based on damagefracture evolution theory. Chinese J. Rock Mechanics and Eng. 26(8): 1588-1594

Ding W-H, Chen H-Q and Zhang J-J 2006 X-ray CT detecting on concrete fracture process under high strain rate. J. Building Structures Supp2: 758-762

Ding W-H, Chen H-Q, Yin XT, Liu S-C and Zhang Jian 2009 Study of key techniques of dynamic loading CT test device for rock-like material. Chinese J. Rock Mechanics and Eng. 28(8): 1620-1628

Garboczi E J 2002 Three-dimensional mathematical analysis of particle shape using X-ray tomography and spherical harmonics: Application to aggregates used in concrete. Cement and Concrete Res. 32(10): $1621-1638$ 
Morgan I L, Ellinger H and Klinksiek R 1980 Examination of concrete by computerized tomography. ACI J. 77(1): 23-27

Suzuki Tetsuya, Ogata Hidehiko, Takada Ryuichi, Aoki Masao and Masayasu Ohtsu 2010 Use of acoustic emission and X-ray computed tomography for damage evaluation of freeze-thawed concrete. Construction and Building Materials 24(12): 2347-2352

Tian Wei, Dang F-N and Chen H-Q 2010 Development and application of the dynamic loading apparatus on CT experiment. Rock and Soil Mechanics 31(1): 329-313

Tian Wei, Dang F-N and Chen H-Q 2011 Research on meso Damage-fracture Divisional Zones of Concrete Based on Uniaxial Compression CT Experiment. J. Hydraul. Eng. 42(8): 124-130

Wang L-B, Jinyoung Park and Fu Y-R 2007 Representation of real particles for DEM simulation using X-ray tomography. Construction and Building Materials 21(2): 338-346

Wong R C K and Chau K T 2005 Estimation of air void and aggregate spatial distributions in concrete under uniaxial compression using computer tomography scanning. Cement and Concrete Research 35(8): $1566-1576$ 